\title{
Extended Noise Analysis Model of CW-type Radar Sensors with IQ Down-Conversion
}

\author{
Vojtech JENIK ${ }^{1}$, Premysl HUDEC ${ }^{1}$, Petr PANEK ${ }^{2}$, \\ ${ }^{1}$ Dept. of Czech Technical University in Prague, Technicka 2, 160 00, Praha 6, Czech Republic \\ ${ }^{2}$ Inst. of Photonics and Electronics, Academy of Sciences of the Czech Republic, Chaberska 57, Prague 8, Czech Republic \\ jenikvoj@fel.cvut.cz
}

Submitted July 19, 2017 / Accepted January 9, 2018

\begin{abstract}
CW-type structures, especially those in the form of small sensors, are amongst the most frequently employed radars in security applications or the automotive industry. The range and operational reliability of these sensors strongly depend upon their noise parameters which this paper examines by extending previously published works and considering all potential inner noise sources to create a complex noise model of the radar family in question here, including commonly used IQ signal processing. The noise sources in question cover local oscillator amplitude-noise and phase-noise, noise from an RF signal path, and noise from a low-frequency signal path. The resulting noise model was verified by practical measurements which included two different local oscillator type, two different low-frequency bandwidths and several different time-delays of the cross-talked signals. In all cases, the agreement between theory and measurement was within the estimated limits. The developed model is applicable for noise analysis and the design of all common $\mathrm{CW}$ type radars.
\end{abstract}

\section{Keywords}

$\mathrm{CW}$ radar, FM-CW radar, $\mathrm{PN}-\mathrm{CW}$ radar, amplitude noise, phase noise, noise analysis, noise model

\section{Introduction}

In recent years, radars based on a continuous wave (CW) structure have risen in importance. Above all, this concerns basic CW, frequency-modulated CW (FM-CW), step-frequency CW (SF-CW), or pseudo-noise CW (PN$\mathrm{CW})$ devices. Their popularity is due to their relative simplicity and the capability of being used as small sensors in many applications (automotive [1-4], medical [5], [6], human detection and rescue [7], [8], others [9]). Nowadays, probably the most important field is the automobile industry where FM-CW units are widely used as low- to mid-range radar sensors. Since the $\mathrm{CW}$-type radars show zero dead-zone and are capable of easily detecting moving targets, they are often also applied as motion detectors in security applications or as missile/projectile detectors in military applications.

In the majority of the applications described above, satisfactory measurement range and the operational reliability of these radars is crucial. To a significant degree, both properties depend upon radar noise behavior. This behavior is influenced by the following physical phenomena: Phase noise of the local oscillator (LO); amplitude noise of the LO; noise from the RF signal path and noise from the low-frequency (LF) signal path. Basic noise analyses of the structures in question can be found, e.g., in [10-12]; [13] and [14] describe the impact of amplitude and the phase noise of the LO on the estimation of target parameters, but none of the literature treats the problem in a comprehensive way. This fact concerns LO amplitude noise in particular as it is usually considered to be of minor importance.

In [15], an attempt to cover the majority of the above described noise sources clearly was published. It touched upon the basic CW structure, considered LO phase noise, the noise of the radio-frequency (RF) and low-frequency (LF) paths, and included a series of practical measurements. But further development revealed phenomena that could not be explained by the theory provided. This paper represents the result of extensive additional research and extends [15] by considering the influences of amplitude noise, as well as providing insight in the noise analysis of structures with IQ signal processing (with IQ down-conversion) which is widely used in modern $\mathrm{CW}$-type radars.

The structure of the paper is as follows: Section 2 evaluates LO noise sources with a focus on IQ signal processing, while Section 3 is dedicated to both phase and amplitude noise measurements of the LOs and presents parameters which are used for calculations later in the paper. Sections 4 and 5 are devoted to comprehensive radar system noise analysis and system noise measurement, respectively, while Section 6 concludes with the results and summarizes the advantages of the new noise model. 


\section{Noise Sources}

CW-type radar sensors include the following major noise sources:

- Noise of linear or quasi-linear circuits in the receiver RF signal path

- Noise of the low frequency (LF) signal path

- Phase-noise of the LO

- Amplitude noise of the LO

Noise analysis of the linear RF signal path and LF signal path is discussed in detail in [15]. The following paragraphs are dedicated to a description of the impact of amplitude and phase noise on CW-type radar behavior. As an improvement to radar noise modeling, IQ signal processing is considered.

\subsection{Phase Noise}

The effect of LO phase noise is strongly influenced by a signal $s_{\mathrm{ct}}(t)$ directly cross-talked from a transmitter (TX) output to a receiver (RX) input (see Fig. 1). The reference and cross-talked signals are defined as [15]:

$$
\begin{gathered}
s_{\mathrm{r}}(t)=A_{\mathrm{r}} \cos \left(2 \pi f_{\mathrm{o}} t+\varphi(t)\right), \\
s_{\mathrm{ct}}(t)=A_{\mathrm{ct}} \cos \left(2 \pi f_{\mathrm{o}}(t-\tau)+\varphi(t-\tau)\right)
\end{gathered}
$$

where $f_{\mathrm{o}}$ stands for LO frequency, $A_{\mathrm{r}}$ and $A_{\mathrm{ct}}$ are amplitudes of reference and cross-talked signals, $\varphi(t)$ represents phase noise and $\tau$ depicts the delay between cross-talked and reference signals.

After down-conversion, such a complex base-band (BB) signal can be described as:

$$
s_{\mathrm{BBP}}(t)=A_{\mathrm{BB}} \exp \left\{\mathrm{j}\left[2 \pi f_{\mathrm{o}} \tau+\Delta \varphi(t, \tau)\right]\right\}
$$

while

$$
\Delta \varphi(t, \tau)=\varphi(t)-\varphi(t-\tau)
$$

represents the phase noise difference, $A_{\mathrm{BB}}$ stands for the amplitude of the signal at the down-converter's output.

Supposing $\Delta \varphi(t, \tau)<<1$, the BB signal can be described using in-phase and quadrature components as:

$$
\begin{aligned}
& s_{\mathrm{BBP} \__{-}}(t) \cong A_{\mathrm{BB}}\left[\cos \left(2 \pi f_{\mathrm{o}} \tau\right)-\Delta \varphi(t, \tau) \sin \left(2 \pi f_{\mathrm{o}} \tau\right)\right], \\
& s_{\mathrm{BBP} \_\mathrm{Q}}(t) \cong A_{\mathrm{BB}}\left[\sin \left(2 \pi f_{\mathrm{o}} \tau\right)-\Delta \varphi(t, \tau) \cos \left(2 \pi f_{\mathrm{o}} \tau\right)\right] .
\end{aligned}
$$

Signals (5) and (6) consist of DC components (described by the first terms in brackets) and noise components. The power spectral density (PSD) of BB noise, caused by LO phase-noise for in-phase (I) and quadrature (Q) components, takes its form according to (7) and (8), respectively:

$$
S_{\mathrm{nP}_{-} \mathrm{I}}(f)=P_{\mathrm{BB}} \sin ^{2}\left(2 \pi f_{\mathrm{o}} \tau\right) S_{\Delta \varphi(\mathrm{t}, \tau)}(f),
$$

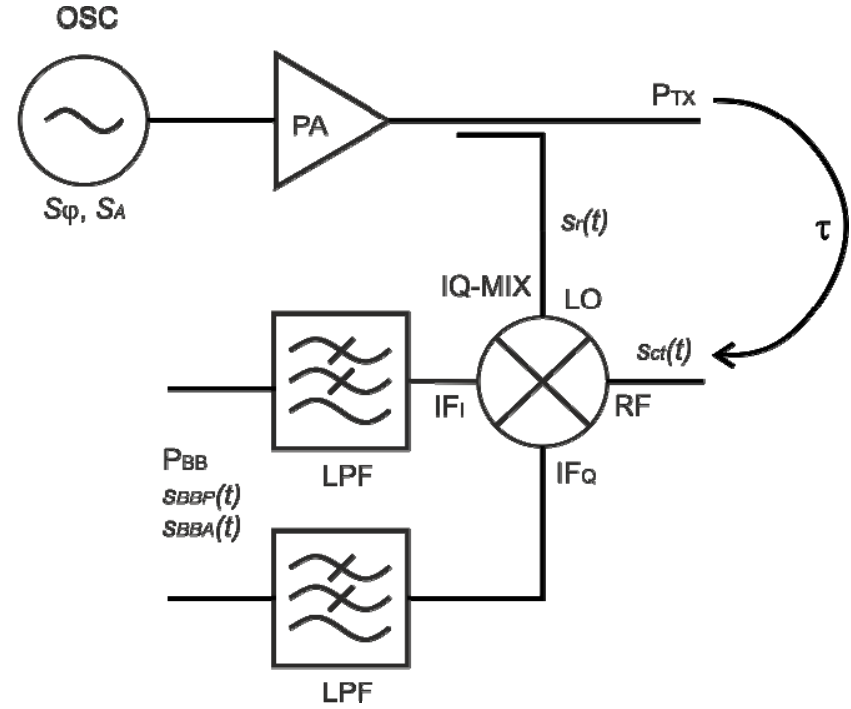

Fig. 1. Phase and amplitude noise propagation through the $\mathrm{CW}$ radar structure.

$$
S_{\mathrm{nP}_{-} \mathrm{Q}}(f)=P_{\mathrm{BB}} \cos ^{2}\left(2 \pi f_{\mathrm{o}} \tau\right) S_{\Delta \varphi(\mathrm{t}, \tau)}(f) \text {. }
$$

In (7) and (8), $P_{\mathrm{BB}}$ stands for the power of signal at the ideal IQ down-converter's outputs (we consider the same amplitude for signals I and Q). According to [13], the spectrum of the phase noise difference takes its form as

$$
S_{\Delta \varphi(t, \tau)}(f)=4 S_{\varphi}(f) \sin ^{2}(\pi f \tau)
$$

where $S_{\varphi}(f)$ represents the normalized PSD of LO phase noise. Using (7) - (9), the PSD of the IQ BB signals can be evaluated as

$$
\begin{aligned}
& S_{\mathrm{nP}_{-} \mathrm{I}}(f)=4 P_{\mathrm{BB}} \sin ^{2}\left(2 \pi f_{\mathrm{o}} \tau\right) \sin ^{2}(\pi f \tau) S_{\varphi}(f), \\
& S_{\mathrm{nP} \_\mathrm{Q}_{-}}(f)=4 P_{\mathrm{BB}} \cos ^{2}\left(2 \pi f_{\mathrm{o}} \tau\right) \sin ^{2}(\pi f \tau) S_{\varphi}(f) .
\end{aligned}
$$

Formulas (10) and (11) show that PSD is a function of time-delay $\tau$ between the reference and the cross-talked signals. The first sine/cosine terms cause oscillations of the noise power in both I and Q channels with $\tau$ changes. It is obvious that the maxima of channel signals $\mathrm{I}$ and $\mathrm{Q}$ are mutually shifted by 90 degrees. Therefore, in the IQ receiver, noise cannot be compensated by a suitable phase shift between the RX input and reference branches. The second sine terms describe changes of phase noise influences for frequencies farther from the DC component. The maxima of the I and Q components (10) and (11) are

$$
S_{\mathrm{nP}}(f)=4 P_{\mathrm{BB}} \sin ^{2}(\pi f \tau) S_{\varphi}(f) .
$$

Assuming the noise bandwidth is known, the total noise power arising from phase noise can be calculated by integrating (12) (only the I-channel formula is presented):

$$
\begin{aligned}
P_{\mathrm{nP}_{-} \mathrm{I}} & =\int_{\mathrm{BW}} S_{\mathrm{nP}_{-} \mathrm{I}}(f, \tau) \mathrm{d} f= \\
& =4 P_{\mathrm{BB}} \sin ^{2}\left(2 \pi f_{\mathrm{o}} \tau\right) \int_{\mathrm{BW}} \sin ^{2}(\pi f \tau) S_{\varphi}(f) \mathrm{d} f .
\end{aligned}
$$


Considering the white phase and white frequency noise components, phase noise PSD can be approximated [15]:

$$
S_{\varphi}(f) \approx \frac{a_{2}}{f^{2}}+a_{0}
$$

Assuming $\pi f \tau<<1$, the resulting noise powers in the I and $\mathrm{Q}$ channels can be expressed as:

$$
\begin{aligned}
& P_{\mathrm{nP}_{-} \mathrm{I}} \approx \\
& 8 P_{\mathrm{BB}} \pi^{2} \tau^{2} \sin ^{2}\left(2 \pi f_{\mathrm{o}} \tau\right)\left[\frac{a_{0}}{3}\left(f_{\mathrm{H}}-f_{\mathrm{L}}\right)^{3}+a_{2}\left(f_{\mathrm{H}}-f_{\mathrm{L}}\right)\right], \\
& P_{\mathrm{nP} \mathrm{Q}_{\mathrm{Q}}} \approx \\
& 8 P_{\mathrm{BB}} \pi^{2} \tau^{2} \cos ^{2}\left(2 \pi f_{\mathrm{o}} \tau\right)\left[\frac{a_{0}}{3}\left(f_{\mathrm{H}}-f_{\mathrm{L}}\right)^{3}+a_{2}\left(f_{\mathrm{H}}-f_{\mathrm{L}}\right)\right] .
\end{aligned}
$$

If the $\pi f \tau<<1$ condition is not satisfied, numerical integration can be used.

\subsection{Amplitude Noise}

The derivation of amplitude noise effects considers the $s_{\mathrm{r}}(t)$ cross-talked signal described by (17), and the $s_{\mathrm{ct}}(t)$ reference signal described by (18). In this case, with respect to $s_{\mathrm{r}}(t), s_{\mathrm{ct}}(t)$ is delayed by $\tau$, and both signals are affected by $A_{\mathrm{n}}(t)$ amplitude noise only. Amplitude noise is a random process with power spectral density $S_{\mathrm{A}}(f)$.

$$
\begin{gathered}
s_{\mathrm{r}}(t)=A_{\mathrm{r}}\left(1-A_{\mathrm{n}}(t)\right) \cos \left(2 \pi f_{\mathrm{o}} t\right), \\
s_{\mathrm{ct}}(t)=A_{\mathrm{ct}}\left(1-A_{\mathrm{n}}(t-\tau)\right) \cos \left(2 \pi f_{\mathrm{o}}(t-\tau)\right) .
\end{gathered}
$$

The complex signal at the output of the mixer can be expressed as follows:

$$
s_{\text {BBA }}(t)=A_{\text {BB }}\left(1-A_{\mathrm{n}}(t)\right)\left(1-A_{\mathrm{n}}(t-\tau)\right) \mathrm{e}^{\mathrm{j}\left(2 \pi f_{\mathrm{o}} \tau\right)} .
$$

The corresponding base-band signal can be described using components I and Q as:

$$
\begin{aligned}
& s_{\mathrm{BBA} \_\mathrm{I}}(t) \cong A_{\mathrm{BB}} \cos \left(2 \pi f_{\mathrm{o}} \tau\right) s_{\mathrm{AA}}(t, \tau), \\
& s_{\mathrm{BBA} \_\mathrm{Q}}(t) \cong A_{\mathrm{BB}} \sin \left(2 \pi f_{\mathrm{o}} \tau\right) s_{\mathrm{AA}}(t, \tau)
\end{aligned}
$$

where

$$
s_{\mathrm{AA}}(t, \tau)=\left(1-A_{\mathrm{n}}(t)\right)\left(1-A_{\mathrm{n}}(t-\tau)\right)
$$

is the amplitude noise product with spectrum $S_{\mathrm{AA}}(f)$.

The PSD of noise, caused by LO amplitude noise for components I and Q, is formed according to (23) and (24), respectively:

$$
\begin{aligned}
& S_{\mathrm{nA} \__{-}}(f)=P_{\mathrm{BB}} \cos ^{2}\left(2 \pi f_{\mathrm{o}} \tau\right) S_{\mathrm{AA}}(f), \\
& S_{\mathrm{nA} \__{-}}(f)=P_{\mathrm{BB}} \sin ^{2}\left(2 \pi f_{\mathrm{o}} \tau\right) S_{\mathrm{AA}}(f) .
\end{aligned}
$$

According to [14], $S_{\mathrm{AA}}$ can be expressed as:

$$
S_{\mathrm{AA}}(f)=4 S_{\mathrm{A}}(f) \cos ^{2}(\pi f \tau)+2 R_{\mathrm{A}}(\tau) \delta(f)
$$

where $R_{\mathrm{A}}(\tau)$ is an autocorrelation of the amplitude noise. The last term in (25) represents a DC component which is filtered out, so it can be omitted. The total power due to oscillator amplitude noise can be calculated using (26).

$$
\begin{aligned}
P_{\mathrm{nA} \_\mathrm{I}} & =\int_{\mathrm{BW}} S_{\mathrm{nAM}}(f) \mathrm{d} f= \\
& =4 P_{\mathrm{BB}} \cos ^{2}\left(2 \pi f_{\mathrm{o}} \tau\right) \int_{\mathrm{BW}} \cos ^{2}(\pi f \tau) S_{\mathrm{A}}(f) \mathrm{d} f .
\end{aligned}
$$

Considering white noise and flicker noise approximation of the oscillator amplitude, the noise spectrum is:

$$
S_{\mathrm{A}}(f) \approx b_{0}+\frac{b_{1}}{f} .
$$

In this formula, $b_{0}$ represents white noise PSD and $b_{1}$ defines the amount of flicker noise. The amplitude noise spectrum is, then, expressed as:

$$
S_{\mathrm{nA} \_\mathrm{I}}(f)=4 P_{\mathrm{BB}} \cos ^{2}\left(2 \pi f_{\mathrm{o}} \tau\right) \cos ^{2}(\pi f \tau)\left(b_{0}+\frac{b_{1}}{f}\right) .
$$

The DSB power of amplitude noise is:

$$
P_{\mathrm{nA}_{-} \mathrm{I}}=8 P_{\mathrm{BB}} \cos ^{2}\left(2 \pi f_{\mathrm{o}} \tau\right) \int_{f_{\mathrm{L}}}^{f_{\mathrm{H}}} \cos ^{2}(\pi f \tau)\left(b_{0}+\frac{b_{1}}{f}\right) \mathrm{d} f .
$$

The integrals presented above do not have analytic solutions. But since radar bandwidth is smaller than $1 \mathrm{GHz}$ with $\tau$ usually being in order of $10^{\circ} \mathrm{ns}$, and, therefore, $\tau f<<1$ in most cases, the second cosines are approximately equal to 1 allowing equations (28) and (29) to be simplified to (30) and (31).

$$
\begin{gathered}
P_{\text {nA_I }} \approx 8 P_{\mathrm{BB}} \cos ^{2}\left(2 \pi f_{\mathrm{o}} \tau\right) \int_{f_{\mathrm{L}}}^{f_{\mathrm{H}}}\left(b_{0}+\frac{b_{1}}{f}\right) \mathrm{d} f= \\
=8 P_{\mathrm{BB}} \cos ^{2}\left(2 \pi f_{\mathrm{o}} \tau\right)\left[b_{0} f+b_{1} \ln (f)\right]_{f_{\mathrm{L}}}^{f_{\mathrm{H}}}, \\
P_{\mathrm{nA} \_\mathrm{Q}} \approx 8 P_{\mathrm{BB}} \sin ^{2}\left(2 \pi f_{\mathrm{o}} \tau\right)\left[b_{0} f+b_{1} \ln (f)\right]_{f_{\mathrm{L}}}^{f_{\mathrm{H}}} .
\end{gathered}
$$

\section{Local Oscillator Noise Measurement}

The practical evaluation of the impact of oscillator noise on radar parameters requires a measurement of LO phase noise and amplitude noise PSD frequency dependences. The measurement of phase noise is described, e.g., in an application note [16], and papers [17-22]. In [18], [19], descriptions of simultaneous amplitude and phase noise measurement are presented. In [23], a more precise amplitude noise measurement method can be found.

Firstly, both phase and amplitude noise measurements were examined on the $11 \mathrm{GHz}$ dielectric resonator oscilla- 
tor (DRO) sample. Subsequently, the same parameters were evaluated for the $11 \mathrm{GHz}$ PLL based LO.

\subsection{DRO Phase Noise Measurement}

The oscillator phase noise spectrum can be evaluated by measuring the instantaneous changes of phase of its output signal. Since ADCs are not capable of handling higher microwave frequencies $(11 \mathrm{GHz}$ in our case), a signal conversion was applied. This approach is well-described in [18], and is based on the subtraction of linear phase dependences from the measured phase time dependence of the acquired signal leaving only phase deviations $\varphi(t)$. The signal evaluated from the phase deviation is equivalent to the frequency shift of the acquired signal without any amplitude modulation. This process can be described as:

$$
\begin{aligned}
s_{\triangle \varphi}(t) & =\exp \left\{\mathrm{j}\left[2 \pi f_{\mathrm{B}} t+\varphi(t)\right]\right\} \exp \left(-2 \mathrm{j} \pi f_{\mathrm{B}} t\right) \\
& =\exp [\mathrm{j} \varphi(t)]
\end{aligned}
$$

where $f_{\mathrm{B}}$ describes beat frequency (frequency of the linear phase change).

The measured DRO phase noise PSD versus offset frequency plot is depicted in Fig. 2. The peaks in measured PSD are caused by using a low beat frequency of approximately $10 \mathrm{kHz}$ in the $\mathrm{LO}$ noise measurement.

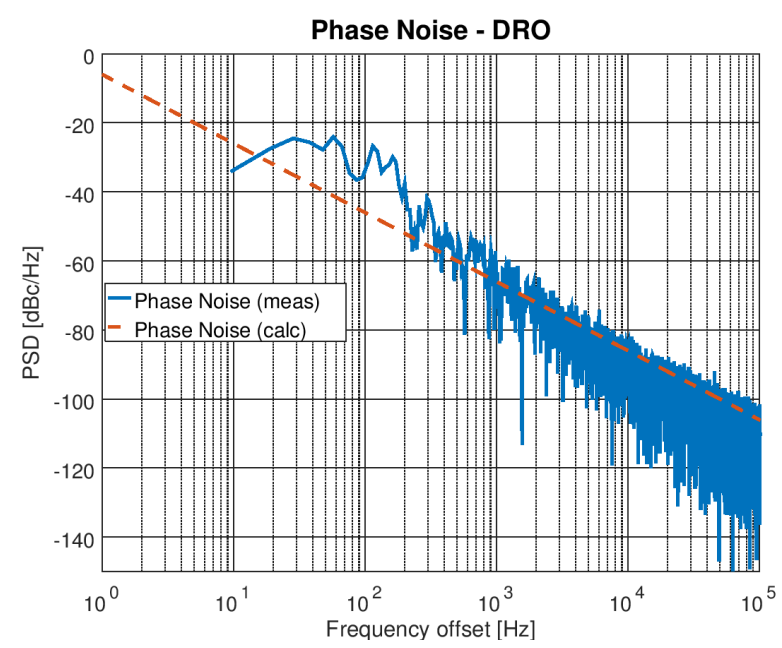

Fig. 2. Measured and approximated PSD due to the phase noise of a DRO-type oscillator at $f_{\mathrm{c}}=11 \mathrm{GHz}$.
According to (14), the resulting coefficients of approximation are $a_{0}=3.2 \times 10^{-14}, a_{2}=0.4 \mathrm{~s}^{-2}$. The $a_{0}$ coefficient corresponds to phase noise far from the carrier. The measurement by a spectrum analyzer results in a noise floor of $-135 \mathrm{dBc} / \mathrm{Hz}$. A separate measurement was necessitated by the relatively high noise floor (around $-125 \mathrm{dBc} / \mathrm{Hz}$ ) of the previously described measurement method caused by the limited number of samples and dynamic range of the ADC used.

To ensure that the measurement is not affected by generator phase noise, an Agilent E8257D generator was used (its phase noise PSD is $-143 \mathrm{dBc} / \mathrm{Hz}$ at $20 \mathrm{kHz}$ carrier offset).

\subsection{DRO Amplitude Noise Measurement}

Since the method described in [18] suffers from insufficient amplitude noise sensitivity, the DRO amplitude noise measurement was performed according to [20]. This correlation method uses two diode detectors with each followed by low frequency amplifier chains, see Fig. 3. Amplitude noise PSD can be calculated using (33).

$$
S_{\mathrm{A}}(f)=\frac{S_{\mathrm{ba}}(f)}{4 k_{\mathrm{a}} k_{\mathrm{b}} P_{\mathrm{a}} P_{\mathrm{b}}} .
$$

In this equation, $S_{\mathrm{ba}}$ represents the cross-spectrum of $a$ and $b$ channels, $k_{\mathrm{a}}$ and $k_{\mathrm{b}}$ stand for detector conversion constants, while $P_{\mathrm{a}}$ and $P_{\mathrm{b}}$ depict corresponding detector input power levels. The efficient suppression of the detectors and amplifying chain noise is a major advantage of this correlation method. To suppress other noise sources, additional averaging was applied in the FFT process used.

For the evaluation of $k_{\mathrm{a}}$ and $k_{\mathrm{b}}$ detector conversion constants, small changes were made to detector input power $P$ around $P_{\mathrm{a}}$ and $P_{\mathrm{b}}$ and the corresponding detected voltages $v_{\mathrm{da}}$ and $v_{\mathrm{db}}$ were read. The detector conversion constants can be calculated as

$$
k_{\mathrm{d} i}=\frac{\mathrm{d} v_{\mathrm{d} i}}{\mathrm{~d} P}, \quad i=\mathrm{a}, \mathrm{b} .
$$

The equation can be modified to:

$$
k_{\mathrm{d} i} P_{i}=\frac{\Delta v_{\mathrm{d} i}}{\Delta P / P_{i}}, \quad i=\mathrm{a}, \mathrm{b} .
$$

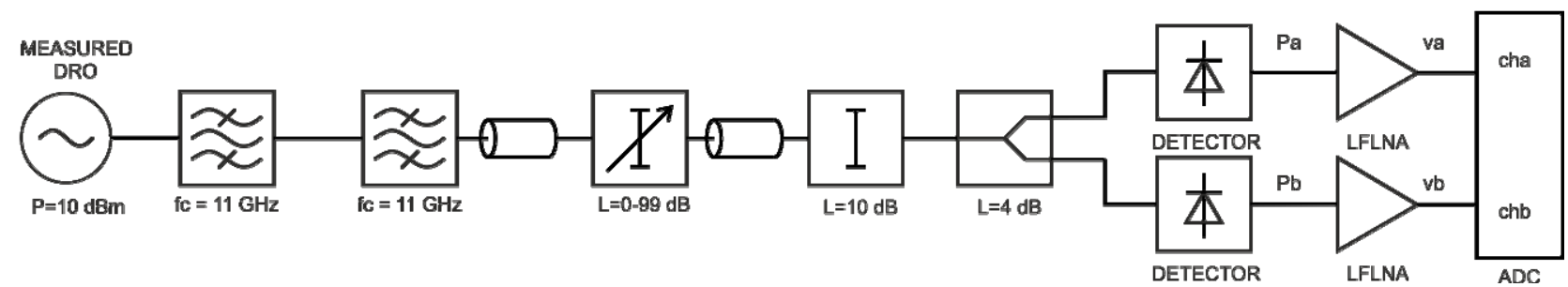

Fig. 3. Measurement setup used for the oscillator amplitude noise measurement. 


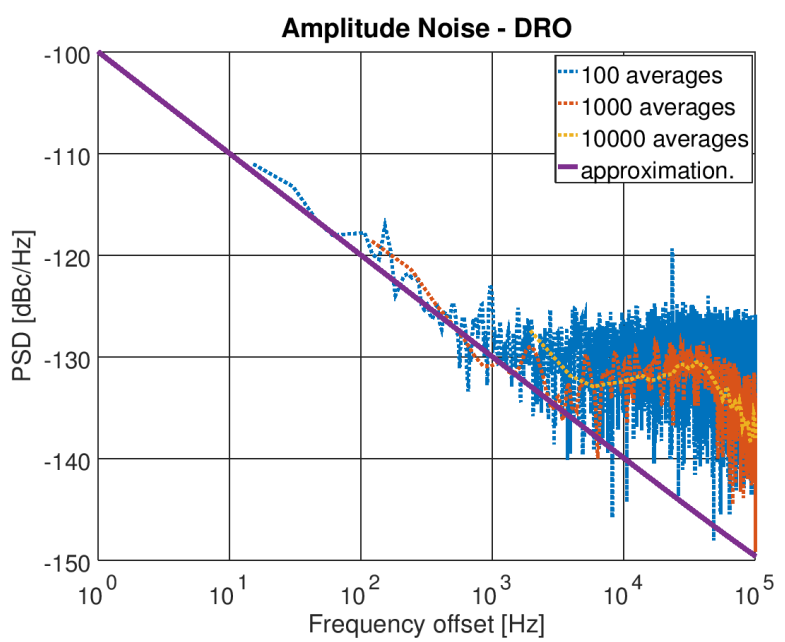

Fig. 4. DRO type $\mathrm{LO}$ at $f_{\mathrm{c}}=11 \mathrm{GHz}$, measured crosscorrelation amplitude noise PSD (100, 1000 and 10000 averages to lower the noise floor) and its approximation.

The $k_{\mathrm{d} i} P_{i}$ product is both easier to measure (relative power measurements are more accurate) and sufficient to calculate the final PSD of amplitude noise.

The cross-spectrum was calculated from signals measured according to Fig. 3 using the FFT. The noise floor of this process was evaluated from the FFT gain and the number of averaged signal samples which was estimated to be around $-145 \mathrm{dBm}$. The real noise floor of amplitude noise is probably much lower; however, it has a negligible impact on the calculated results of amplitude noise.

The resulting amplitude noise PSD is depicted in Fig. 4. The spectrum was approximated by (27) with coefficients $b_{0}=3.2 \times 10^{-15}, b_{1}=10^{-10} \mathrm{~s}^{-1}$.

\subsection{PLL-based Oscillator Noise Measurement}

The noise parameters of the PLL-based LO were measured in the same way as the DRO-type LO. The obtained PSD of the phase noise is depicted in Fig. 5. However, amplitude noise was below the limits of the measurement conditions, so it was considered to be a constant value of $-145 \mathrm{dBc} / \mathrm{Hz}$. This corresponds to coefficients $b_{0}=3.2 \times 10^{-15}, b_{1}=0 \mathrm{~s}^{-1}$ in (27).

Since PLLs suppress phase noise at lower offset frequencies, the noise spectrum of these oscillators cannot be approximated by (14). The phase noise was approximated by (36) instead.

$$
S_{\varphi}(f) \approx \begin{cases}a_{01}, & f \leq f_{1} \\ \frac{a_{3}}{f^{3}}+a_{0}, & f \geq f_{1}\end{cases}
$$

In this formula, $a_{01}$ represents noise power density in the active PLL bandwidth, while $f_{1}$ stands for the corresponding cutoff frequency. Approximating coefficients are

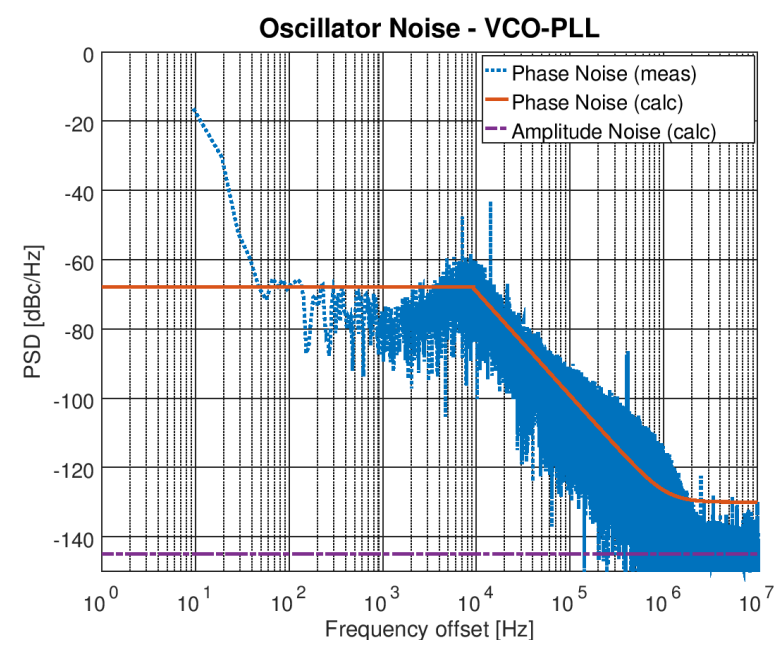

Fig. 5. PLL-type LO, measured PSD of phase noise, approximated PSD of phase and amplitude noise.

$a_{01}=1.6 \times 10^{-7}, f_{1}=9280 \mathrm{~Hz}, a_{0}=10^{-13}, a_{3}=1.2 \times 10^{5} \mathrm{~s}^{-3}$. The resulting amplitude and phase noise PSD plots are presented in Fig. 5.

\section{System Noise Analysis}

Figure 6 shows a block diagram of the measurement setup used to evaluate the overall noise behavior of the CW-type radar sensors in question. It consists of the basic radar structure common to all $\mathrm{CW}$-type versions and additional components needed for the performed tests. Radar TX consists of the DRO, or PLL-type, the LO, a PA power amplifier and a power splitter dividing the signal to the TX output and reference branch. The RX branch consists of a coherent IQ down-converter fed by the reference signal from one output of the TX splitter followed by the LF filtering-amplifying branches.

To achieve the required time-delay values to distinguish between amplitude and phase noise, the test part of the measurement setup includes a set of co-axial cable sections of different lengths (1-30 m), and a variable length strip-line (to measure small time-delay differences). A set of RF amplifiers is used to compensate for the high insertion loss of the concerned time-delay components. The entire test circuit affects the noise calculation as specified below.

According to Fig. 6, the $P_{\mathrm{nRF}}$ total noise power generated by linear RF components at the filtered IQ demodulator outputs (considering the output LP filters and both sidebands) can be expressed by (37).

$$
P_{\mathrm{nRF}}=2 P_{\mathrm{nRFSSB}}=2\left(P_{\mathrm{nRFT}} G_{\mathrm{SYS}}+P_{\mathrm{nRFSYS}}\right) \text {. }
$$

In this formula, $P_{\mathrm{nRFT}}$ represents noise power at the output of the test circuit and $P_{\text {nRFSYs }}$ and $G_{\text {SYS }}$ stand for output noise power and gain of the RF radar circuits, respectively. Noise power of the test circuit is further expressed in (38): 


$$
\begin{aligned}
& P_{\mathrm{nRFT}}= \\
& k T_{0} B_{\mathrm{n}}\left(L_{\mathrm{DEL}}-1\right) \frac{G_{\mathrm{AMP}}}{L_{\mathrm{DEL}}}+k T_{0} B_{\mathrm{n}}\left(F_{\mathrm{AMP}}-1\right) G_{\mathrm{AMP}} .
\end{aligned}
$$

In this equation, $B_{\mathrm{n}}$ stands for noise bandwidth; $L_{\mathrm{DEL}}$ incorporates the loss of the inserted cable and variable length strip line, together with the attenuators used for cable loss compensation. $G_{\mathrm{AMP}}$ and $F_{\mathrm{AMP}}$ stand for the total gain and noise figure of the amplifiers used.

Noise power of the RF radar circuits takes mixer gain $G_{\mathrm{MIX}}$, mixer noise figure $F_{\mathrm{MIX}}$ and losses in the low pass filter $L_{\mathrm{LPF}}$ into account:

$$
\begin{aligned}
& P_{\mathrm{nRFSYS}}= \\
& k T_{0} B_{\mathrm{n}} \frac{\left(F_{\mathrm{MIX}}-1\right) G_{\mathrm{MIX}}}{L_{\mathrm{LPF}}}+k T_{0} B_{\mathrm{n}} \frac{\left(L_{\mathrm{LPF}}-1\right)}{L_{\mathrm{LPF}}}, \\
& G_{\mathrm{SYS}}=\frac{G_{\mathrm{MIX}}}{L_{\mathrm{LPF}}} .
\end{aligned}
$$

The contribution of the amplitude and phase noise of the local oscillator can be evaluated using (30) and (15), respectively. Signal power $P_{\mathrm{BB}}$ from (7-8) and (23-24) at the LPF output is expressed by (41):

$$
P_{\mathrm{BB}}=P_{\mathrm{TX}} \frac{G_{\mathrm{AMP}}}{L_{\mathrm{CAB}}} G_{\mathrm{SYS}} .
$$

From [15], the noise of the LF signal path $P_{\mathrm{nLF}}$ is referred to $Z_{0}$ impedance at the filtered down-converter outputs. In these planes (behind LPFs), $50 \mathrm{ohm}$ impedance match paths usually end and are followed by voltage amplifiers which is why it can be advantageous to refer the LF noise of LF amplifiers to their inputs. It can be expressed using formulas describing LF amplifier noise voltages and currents:

$$
V_{\mathrm{nLF}}=\sqrt{B_{\mathrm{n}}\left[e_{\mathrm{n}}^{2}+e_{\mathrm{nR}}^{2}+\left(i_{\mathrm{n}} R_{\mathrm{eq}}\right)^{2}\right]} .
$$

In this formula, $e_{\mathrm{n}}$ stands for the input noise voltage of the given $\mathrm{OA}$ (the value is stated in $\left[\mathrm{nV} \cdot \mathrm{Hz}^{-1 / 2}\right]$ ) and $i_{\mathrm{n}}$ represents the input noise current of the given OA (the value is stated in $\left.\left[\mathrm{pA} \cdot \mathrm{Hz}^{-1 / 2}\right]\right) . e_{\mathrm{nR}}$ is the noise voltage generated by the feedback resistors.

This plane (marked A in Fig. 6) is defined as the noise calculation plane where all inner noise sources can be summed. In this plane, $P_{\mathrm{nT}}$ total noise power can be calculated as:

$$
P_{\mathrm{nT}}=P_{\mathrm{nA}}+P_{\mathrm{nP}}+P_{\mathrm{nRF}}+P_{\mathrm{nLF}} .
$$

The relation between $V_{i}$ noise voltage and $P_{i}$ noise power is shown in this formula (44).

$$
P_{\mathrm{nT}}=\frac{V_{\mathrm{nT}}^{2}}{R_{0}} .
$$

A list including real values of parameters, used in the measurement setup and for calculations, is presented in Tab. 1. Calculations were made for 2 different $B_{\mathrm{n}}$ noise bandwidths reaching 9 and $159 \mathrm{kHz}$, which corresponds to the processing of both narrowband and more broadband CW-type radar signals.

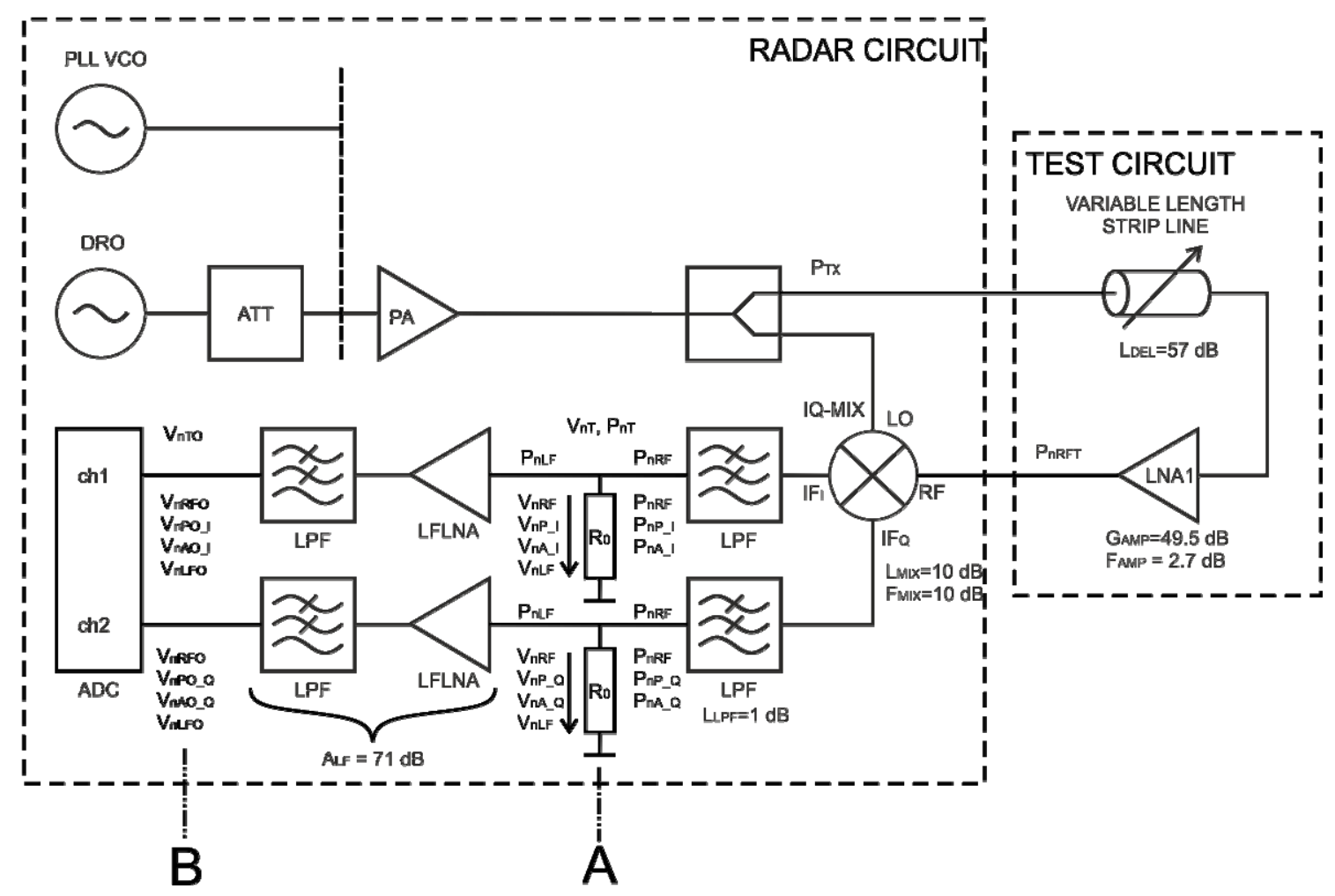

Fig. 6. Block diagram of the measurement setup. Variables are placed close to the points they are related to. 


\begin{tabular}{|c|c|c|c|}
\hline Parameter & Description & Value & Unit \\
\hline$T_{0}$ & ambient temp. & 300 & $\mathrm{~K}$ \\
\hline$f_{\mathrm{L}}$ & low frequency limit (BB) & 1 & $\mathrm{kHz}$ \\
\hline$f_{\mathrm{H}}$ & high frequency limit (BB) & $10 / 160$ & $\mathrm{kHz}$ \\
\hline$B_{\mathrm{n}}$ & noise bandwidth & $9 / 159$ & $\mathrm{kHz}$ \\
\hline$P_{\mathrm{TX}}$ & $\begin{array}{c}\text { transmitted power } \\
\text { (DRO/PLL VCO) }\end{array}$ & $13 / 19$ & $\mathrm{dBm}$ \\
\hline$L_{\mathrm{DEL}}$ & delay line total loss & 57 & $\mathrm{~dB}$ \\
\hline$G_{\mathrm{AMP}}$ & gain of compensating amps & 49.5 & $\mathrm{~dB}$ \\
\hline$F_{\mathrm{AMP}}$ & $\begin{array}{c}\text { noise figure } \\
\text { of compensating amps }\end{array}$ & 2.7 & $\mathrm{~dB}$ \\
\hline$G_{\mathrm{MIX}}$ & mixer gain & 10 & $\mathrm{~dB}$ \\
\hline$F_{\mathrm{MIX}}$ & mixer noise figure & 10 & $\mathrm{~dB}$ \\
\hline$L_{\mathrm{LPF}}$ & LP filter loss & 1 & $\mathrm{~dB}$ \\
\hline$A_{\mathrm{LF}}$ & voltage gain of LF block & 71 & $\mathrm{~dB}$ \\
\hline
\end{tabular}

Tab. 1. Parameters of measurement setup components.

\begin{tabular}{|c|c|c|c|}
\hline $\boldsymbol{B}_{\mathbf{n}}[\mathrm{kHz}]$ & $\boldsymbol{P}_{\mathbf{n R F}}[\mathrm{W}]$ & $\boldsymbol{V}_{\mathbf{n R F}}[\mathrm{mV}]$ & $\mathbf{V}_{\mathbf{n R F O}}[\mathrm{mV}]$ \\
\hline 9 & $0.99 \mathrm{e}-12$ & $7.0 \mathrm{e}-3$ & 25.0 \\
\hline 159 & $17.5 \mathrm{e}-12$ & $29.6 \mathrm{e}-3$ & 105 \\
\hline
\end{tabular}

Tab. 2. Calculated noise parameters caused by RF signal path.

\begin{tabular}{|c|c|c|}
\hline $\boldsymbol{B}_{\mathrm{n}}[\mathrm{kHz}]$ & $\boldsymbol{V}_{\mathrm{nLF}}[\mathrm{mV}]$ & $\boldsymbol{V}_{\mathrm{nLFO}}[\mathrm{mV}]$ \\
\hline 9 & $1.2 \mathrm{e}-4$ & 0.5 \\
\hline 159 & $5.3 \mathrm{e}-4$ & 1.7 \\
\hline
\end{tabular}

Tab. 3. Calculated noise voltages corresponding to the LF signal path.

\begin{tabular}{|c|c|c|c|c|c|c|}
\hline $\begin{array}{c}\boldsymbol{B}_{\mathbf{n}} \\
{[\mathrm{kHz}]}\end{array}$ & $\begin{array}{c}\boldsymbol{\tau} \\
{[\mathrm{ns}]}\end{array}$ & $\begin{array}{c}\boldsymbol{V}_{\text {nP }} \\
{[\mathrm{mV}]}\end{array}$ & $\begin{array}{c}\boldsymbol{V}_{\text {nA }} \\
{[\mathrm{mV}]}\end{array}$ & $\begin{array}{c}\boldsymbol{V}_{\text {nPO }} \\
{[\mathrm{mV}]}\end{array}$ & $\begin{array}{c}\boldsymbol{V}_{\text {nAO }} \\
{[\mathrm{mV}]}\end{array}$ & $\begin{array}{c}\boldsymbol{V}_{\text {nTo }} \\
{[\mathrm{mV}]}\end{array}$ \\
\hline \multirow{4}{*}{9} & 5 & $0.3 \mathrm{e}-3$ & $5.4 \mathrm{e}-3$ & 1.1 & 19.2 & 31.4 \\
\cline { 2 - 7 } & 48.1 & $3.0 \mathrm{e}-3$ & $5.4 \mathrm{e}-3$ & 10.8 & 19.2 & 33.3 \\
\cline { 2 - 7 } & 96.1 & $6.1 \mathrm{e}-3$ & $5.4 \mathrm{e}-3$ & 21.5 & 19.2 & 38.2 \\
\cline { 2 - 7 } & 144 & $9.1 \mathrm{e}-3$ & $5.4 \mathrm{e}-3$ & 32.3 & 19.2 & 45.1 \\
\hline \multirow{4}{*}{159} & 5 & $1.3 \mathrm{e}-3$ & $10.7 \mathrm{e}-3$ & 4.7 & 37.9 & 112 \\
\cline { 2 - 7 } & 48.1 & $12.8 \mathrm{e}-3$ & $10.7 \mathrm{e}-3$ & 45.4 & 37.9 & 120 \\
\cline { 2 - 7 } & 96.1 & $25.6 \mathrm{e}-3$ & $10.7 \mathrm{e}-3$ & 90.7 & 37.9 & 144 \\
\cline { 2 - 7 } & 144 & $38.3 \mathrm{e}-3$ & $10.7 \mathrm{e}-3$ & 136 & 37.9 & 176 \\
\hline
\end{tabular}

Tab. 4. DRO-based radar - Calculated noise voltages caused by LO amplitude and phase noise as a function of time-delay and frequency bandwidth. The total noise voltage is presented in the last column.

\begin{tabular}{|c|c|c|c|c|c|c|}
\hline $\begin{array}{c}\boldsymbol{B}_{\mathbf{n}} \\
{[\mathrm{kHz}]}\end{array}$ & $\begin{array}{c}\boldsymbol{\tau} \\
{[\mathrm{ns}]}\end{array}$ & $\begin{array}{c}\boldsymbol{V}_{\mathbf{n P}} \\
{[\mathrm{mV}]}\end{array}$ & $\begin{array}{c}\boldsymbol{V}_{\mathbf{n A}} \\
{[\mathrm{mV}]}\end{array}$ & $\begin{array}{c}\boldsymbol{V}_{\mathbf{n P O}} \\
{[\mathrm{mV}]}\end{array}$ & $\begin{array}{c}\boldsymbol{V}_{\mathbf{n A O}} \\
{[\mathrm{mV}]}\end{array}$ & $\begin{array}{c}\boldsymbol{V}_{\mathbf{n T O}} \\
{[\mathrm{mV}]}\end{array}$ \\
\hline \multirow{5}{*}{9} & 5 & $2.4 \mathrm{e}-3$ & $3.6 \mathrm{e}-3$ & 8.5 & 12.8 & 29.3 \\
\cline { 2 - 7 } & 48.1 & $23.0 \mathrm{e}-3$ & $3.6 \mathrm{e}-3$ & 81.5 & 12.8 & 86.2 \\
\cline { 2 - 7 } & 96.1 & $45.9 \mathrm{e}-3$ & $3.6 \mathrm{e}-3$ & 163 & 12.8 & 126 \\
\cline { 2 - 7 } & 144 & $68.8 \mathrm{e}-3$ & $3.6 \mathrm{e}-3$ & 244 & 12.8 & 246 \\
\hline \multirow{4}{*}{159} & 5 & $6.5 \mathrm{e}-3$ & $15.1 \mathrm{e}-3$ & 23.0 & 53.6 & 120 \\
\cline { 2 - 7 } & 48.1 & $62.4 \mathrm{e}-3$ & $15.1 \mathrm{e}-3$ & 221 & 53.6 & 251 \\
\cline { 2 - 7 } & 96.1 & $125 \mathrm{e}-3$ & $15.1 \mathrm{e}-3$ & 445 & 53.6 & 460 \\
\cline { 2 - 7 } & 144 & $188 \mathrm{e}-3$ & $15.1 \mathrm{e}-3$ & 666 & 53.6 & 677 \\
\hline
\end{tabular}

Tab. 5. PLL-based radar - Calculated noise voltages caused by LO amplitude and phase noise as a function of timedelay and frequency bandwidth. The total noise voltage is presented in the last column.

The results of the calculations of the noise parameters caused by the RF signal path are summarized in Tab. 2. To enable a comparison with the measured results, the $V_{\mathrm{nRF}}$ and $V_{\mathrm{nRFO}}$ filtered noise voltages at the noise calculation plane and at the ADC input, respectively, are also presented.
Noise voltages corresponding to the LF signal path are presented in Tab. 3. $V_{\mathrm{nLF}}$ stands for LF circuit noise recalculated to point A in Fig. 6, $V_{\text {nLFO }}$ represents a voltage of the same noise at point B.

Time-delay dependences of noise voltages are presented in Tab. 4 and 5 (calculations) and Fig. 7 to 10 (calculations and measurements). Tables 4 and 5 show the values of noise voltages corresponding to radar sensors equipped with DRO- and PLL-based LOs, respectively. The partial $V_{\mathrm{nP}}, V_{\mathrm{nA}}$ voltages refer to calculation plane A (Fig. 6) and the $V_{\mathrm{nPO}}, V_{\mathrm{nAO}}$ voltages correspond to the ADC inputs (calculation plane B). $V_{\mathrm{nTO}}$ values stand for the maximum noise voltages at the ADC inputs when considering all noise sources (calculation plane B).

\section{System Noise Measurement Results}

Radar system noise measurements were performed as in the setup shown in Fig. 6. To eliminate LO noise, measurements with a disconnected cable (no crosstalk) were also performed. RMS noise voltages were calculated from the data acquired by AD converters. Applying digital filtering enabled noise voltages to be calculated separately for each considered $B_{\mathrm{n}}$ frequency bandwidth. To prevent aliasing in the $\mathrm{AD}$ converters, analog low-pass filters with a $160 \mathrm{kHz}$ cutoff frequency were used.

All measured values for structures equipped with DRO- and PLL-based LOs are summarized in Tab. 6 and 7. The calculated values are also provided for the sake of comparison. Slightly different values for the I and Q branches can be explained by the slightly different gains in the I and Q branches, and by the mixer amplitude imbalance.

To provide better insight into CW-type radar noise behavior, the calculated and measured results are also presented as functions of time-delay $\tau$ in Fig. 7 to 10 . The measured noise voltages correspond to the $V_{\mathrm{nT}}$ total radar noise voltage, and were evaluated for four discrete $\tau$ values (5, 48.1, 96.1 and $144 \mathrm{~ns})$ created by combining co-axial cable sections and a variable phase-shifter. The $V_{\mathrm{nT}}$ voltages are plotted separately for the I and Q branches. All graphs also include estimated lower and upper $V_{\mathrm{nT}}$ limits. The limits were evaluated with respect to variations of insertion loss of the variable phase shifter used, mixer amplitude imbalance, amplitude imbalance of low frequency circuits and system gain uncertainties caused by system interconnects. To facilitate the understanding of the resulting noise voltage dependences, the graphs also include dependences of the separate phase-noise, amplitudenoise and LF noise components.

The presented results show that the noise behaviors of the CW-type radars equipped with DRO and PLL LOs are notably different. The DRO-based units show relatively high amplitude noise, are independent of $\tau$, and are only a weak function of the $B_{\mathrm{n}}$ noise bandwidth above $1 \mathrm{kHz}$. In 
the case of narrowband systems and low $\tau$ values, it can become a dominant source of noise. It can also be important in the case of low RF noise.

\begin{tabular}{|c|c|c|c|c|}
\hline $\boldsymbol{B}_{\mathbf{n}}[\mathrm{kHz}]$ & $\boldsymbol{\tau}[\mathrm{ns}]$ & $\boldsymbol{V}_{\text {nMO_I }}[\mathrm{mV}]$ & $\boldsymbol{V}_{\text {nMO_Q }}[\mathrm{mV}]$ & $\boldsymbol{V}_{\text {nTO }}[\mathrm{mV}]$ \\
\hline \multirow{4}{*}{9} & 5 & 29.5 & 31.1 & 31.4 \\
\cline { 2 - 5 } & 48.1 & 31.2 & 32.2 & 33.3 \\
\cline { 2 - 5 } & 96.1 & 35.1 & 35.4 & 38.2 \\
\cline { 2 - 5 } & 144 & 43.6 & 41.0 & 45.1 \\
\hline \multirow{4}{*}{159} & 5 & 118 & 128 & 112 \\
\cline { 2 - 5 } & 48.1 & 121 & 133 & 120 \\
\cline { 2 - 5 } & 96.1 & 145 & 159 & 144 \\
\cline { 2 - 5 } & 144 & 169 & 180 & 176 \\
\hline 9 & no crosstalk & 23.7 & 23.3 & 25.0 \\
\hline 159 & no crosstalk & 125 & 135 & 105 \\
\hline
\end{tabular}

Tab. 6. DRO-based radar - Measured noise voltages as functions of $\tau$ and filter bandwidths $B_{\mathrm{n}}$ in comparison with theoretical values.

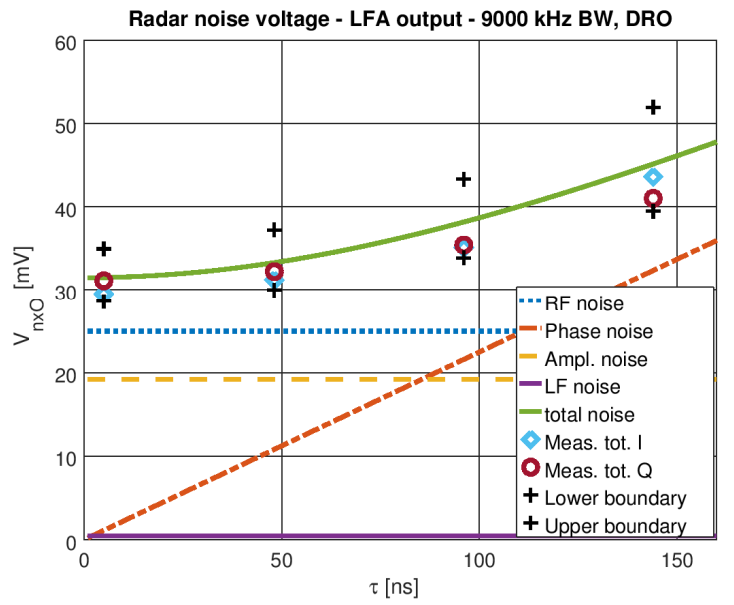

Fig. 7. DRO-based radar - calculated and measured noise voltages at the ADC input (in the following order: $\left.V_{\text {nRFo }}, V_{\text {nPO }}, V_{\text {nAO }}, V_{\text {nLFO }}, V_{\text {nTO }}, V_{\text {nMO_I }}, V_{\text {nMO_Q }}\right)$ at a bandwidth of 1-10 kHz. Lower and upper boundaries represent the expected deviation of the total noise voltage $V_{\text {nTO }}$.

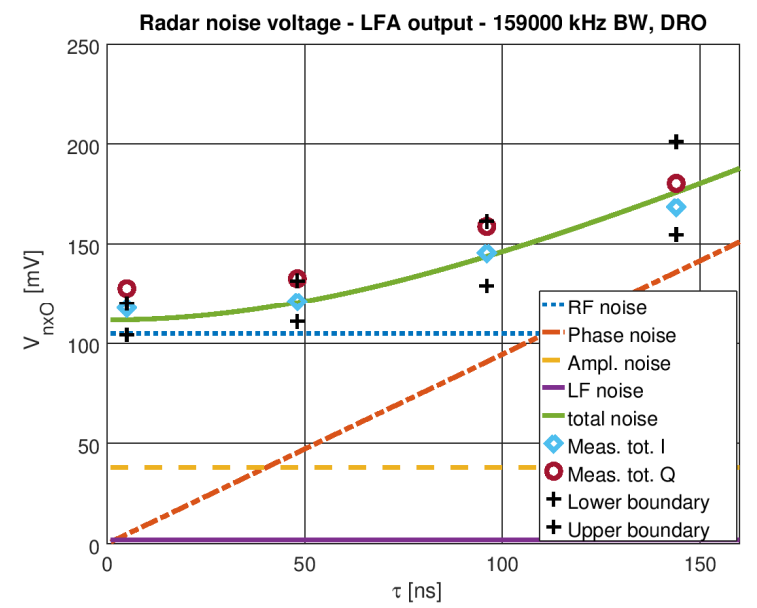

Fig. 8. DRO-based radar - calculated and measured noise voltages at the ADC input (in the following order: $V_{\mathrm{nRFO}}$ $V_{\text {nPO }}, V_{\text {nAO }}, V_{\text {nLFO }}, V_{\text {nTO }}, V_{\text {nMO_I }}, V_{\text {nMO_Q }}$ ) at a bandwidth of $1-160 \mathrm{kHz}$. Lower and upper boundaries represent the expected deviation of the total noise voltage $V_{\text {nTO }}$.

\begin{tabular}{|c|c|c|c|c|}
\hline $\boldsymbol{B}_{\mathbf{n}}[\mathrm{kHz}]$ & $\boldsymbol{\tau}[\mathrm{ns}]$ & $\boldsymbol{V}_{\text {nMO_I }}[\mathrm{mV}]$ & $\boldsymbol{V}_{\text {nMO_Q }}[\mathrm{mV}]$ & $\boldsymbol{V}_{\text {nTO }}[\mathrm{mV}]$ \\
\hline \multirow{4}{*}{9} & 5 & 26.8 & 26.0 & 29.3 \\
\cline { 2 - 5 } & 48.1 & 91.1 & 79.5 & 86.2 \\
\cline { 2 - 5 } & 96.1 & 173 & 157 & 126 \\
\cline { 2 - 5 } & 144 & 224 & 213 & 246 \\
\hline \multirow{4}{*}{159} & 5 & 110 & 120 & 120 \\
\cline { 2 - 5 } & 48.1 & 248 & 264 & 251 \\
\cline { 2 - 5 } & 96.1 & 468 & 483 & 460 \\
\cline { 2 - 5 } & 144 & 625 & 642 & 677 \\
\hline 9 & no crosstalk & 23.7 & 23.3 & 25.0 \\
\hline 159 & no crosstalk & 125 & 135 & 105 \\
\hline
\end{tabular}

Tab. 7. PLL-based radar - measured noise voltages as functions of $\tau$ and filter bandwidths $B_{\mathrm{n}}$ in comparison with theoretical values.

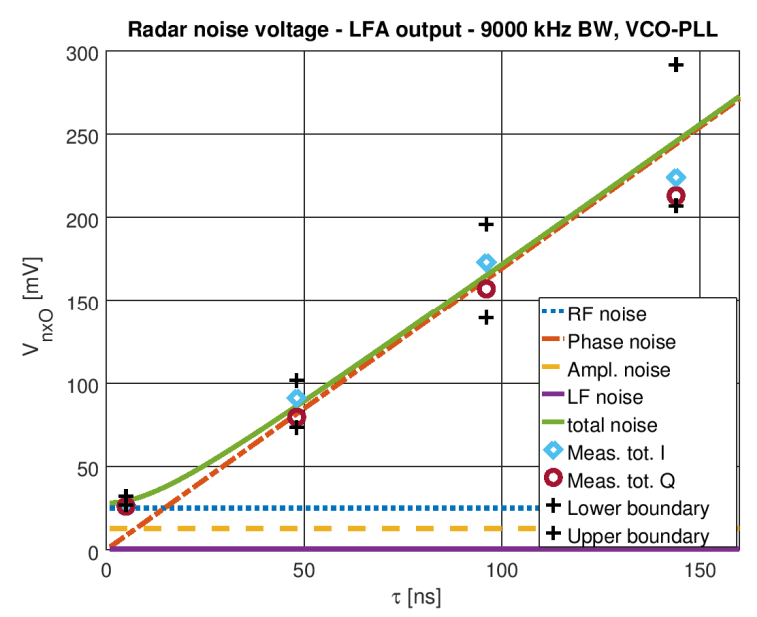

Fig. 9. PLL-based radar - calculated and measured noise voltages at the ADC input (in the following order: $\left.V_{\text {nRFo }}, V_{\text {nPO }}, V_{\text {nAO }}, V_{\text {nLFO }}, V_{\text {nTO }}, V_{\text {nMO_I }}, V_{\text {nMO_Q }}\right)$ at a bandwidth of 1-10 kHz. Lower and upper boundaries represent the expected deviation of the total noise voltage $V_{\text {nTO. }}$.

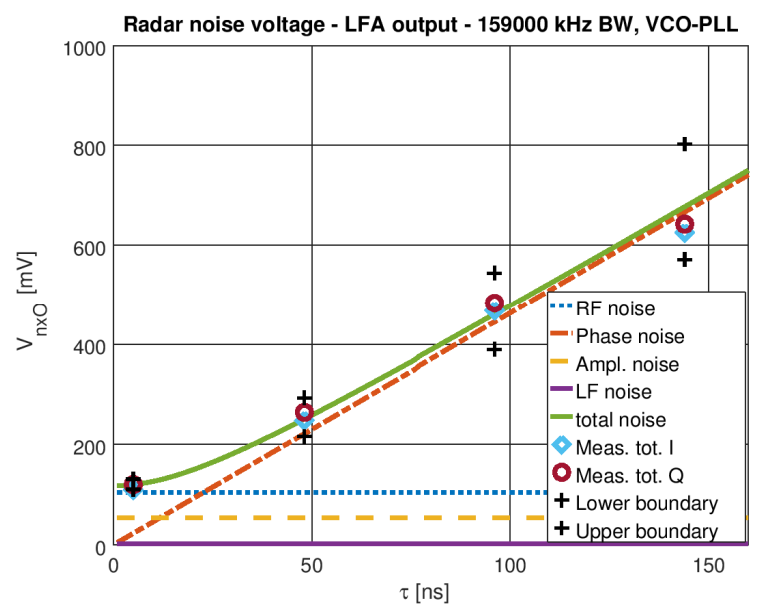

Fig. 10 PLL-based radar, calculated and measured noise voltages at the ADC input (in the following order: $\left.V_{\text {nRFO }}, V_{\text {nPO }}, V_{\text {nAO }}, V_{\text {nLFO }}, V_{\text {nTO }}, V_{\text {nMO I }}, V_{\text {nMO Q }}\right)$ at a bandwidth of $1-160 \mathrm{kHz}$. Lower and upper boundaries represent the expected deviation of the total noise voltage $V_{\text {nTO }}$

The PLL-based units show extremely low amplitude noise and their noise behavior is dominated by RF noise and phase noise. Both radar types used in the tests evince 
negligible LF noise which is caused by the fact that both measurement setups employ low-noise LF amplifiers based on LT1028 operational amplifiers. These ICs are costly but rank amongst the best available low-noise amplifiers with $0.9 \mu \mathrm{V} / \sqrt{\mathrm{Hz}}$ input noise voltage. Using less expensive and lower quality OAs can lead to significantly higher LF noise.

\section{Conclusion}

The operation of CW-type radars is influenced by noise to a significant degree. This work yields a detailed analysis of all inner-noise sources in a structure with IQ signal processing. The considered noise sources include LO phase-noise, LO amplitude noise, noise of linear, or quasi-linear, components in the RF signal path, and the noise of the LF signal path. Based on a detailed description of all these sources, a complex radar noise model has been created and verified. During practical measurements, structures equipped with two different LOs have been tested. In addition, significantly different time-delay $\tau$ values between received and reference signals, and base-band $B_{\mathrm{n}}$ bandwidths have been considered.

The structure employing the DRO-based LO shows high amplitude noise and has a dominant influence in the case of the narrow $B_{\mathrm{n}}$ bandwidth, featuring a low timedelay $\tau$ and low noise from the RF path. Since the PLLbased LOs show substantially lower amplitude noise, phase noise and RF noise represent the major noise sources in these radar structures. The performed measurements confirm that the developed noise model corresponds well to real radar circuits. The developed model can describe noise behavior of both DRO- and PLL-based LOs, of both narrowband and wideband systems, and covers wide ranges of time-delay $\tau$ values. It can contribute to more precise CWtype radar design resulting in more reliable operations.

\section{Appendix}

Calculated and measured noise parameters:

\begin{tabular}{|c|c|l|}
\hline Parameter & Unit & \multicolumn{1}{|c|}{ Description } \\
\hline$P_{\mathrm{nRF}}$ & $\mathrm{W}$ & $\begin{array}{l}\text { Calculated DSB noise power generated by } \\
\text { linear and quasi-linear components at the } \\
\text { LNLFA input }\end{array}$ \\
\hline$V_{\mathrm{nRF}}$ & $\mathrm{V}$ & $\begin{array}{l}\text { Calculated noise voltage at the LNLFA input } \\
\text { corresponding to } P_{\mathrm{nRF}}\end{array}$ \\
\hline$V_{\mathrm{nRFO}}$ & $\mathrm{V}$ & $\begin{array}{l}\text { Noise voltage at the ADC input corresponding } \\
\text { to } P_{\mathrm{nRF}}\end{array}$ \\
\hline$P_{\mathrm{nP}-\mathrm{I}}$ & $\mathrm{W}$ & $\begin{array}{l}\text { Calculated noise power at the LNLFA input in } \\
\text { I channel resulting from the local oscillator } \\
\text { phase noise }(\tau \text { dependence) }\end{array}$ \\
\hline$P_{\mathrm{nP}-}$ & $\mathrm{W}$ & $\begin{array}{l}\text { Calculated noise power at the LNLFA input in } \\
\text { Q channel resulting from the local oscillator } \\
\text { phase noise }(\tau \text { dependence) }\end{array}$ \\
\hline$P_{\mathrm{nP}}$ & $\mathrm{W}$ & $\begin{array}{l}\text { Calculated maximum value of the noise power } \\
\text { at the LNLFA input resulting from the local } \\
\text { oscillator phase-noise }\end{array}$ \\
\hline
\end{tabular}

\begin{tabular}{|c|c|l|}
\hline$V_{\mathrm{nP}}$ & $\mathrm{V}$ & $\begin{array}{l}\text { Calculated maximum value of the noise } \\
\text { voltage at the LNLFA input resulting from the } \\
\text { local oscillator phase-noise }\end{array}$ \\
\hline$V_{\mathrm{nPO}}$ & $\mathrm{V}$ & $\begin{array}{l}\text { Calculated maximum value of the noise } \\
\text { voltage at the ADC input resulting from the } \\
\text { local oscillator phase-noise }\end{array}$ \\
\hline$P_{\mathrm{nA} \_\mathrm{I}}$ & $\mathrm{W}$ & $\begin{array}{l}\text { Calculated noise power at the LNLFA input in } \\
\text { I channel resulting from the local oscillator am- } \\
\text { plitude noise ( } \tau \text { dependence) }\end{array}$ \\
\hline$P_{\mathrm{nA} \_\mathrm{Q}}$ & $\mathrm{W}$ & $\begin{array}{l}\text { Calculated noise power at the LNLFA input in } \\
\text { Q channel resulting from the local oscillator } \\
\text { amplitude noise }(\tau \text { dependence) }\end{array}$ \\
\hline$P_{\mathrm{nA}}$ & $\mathrm{W}$ & $\begin{array}{l}\text { Calculated maximum value of the noise power } \\
\text { at the LNLFA input resulting from the local } \\
\text { oscillator amplitude-noise }\end{array}$ \\
\hline$V_{n A}$ & $\mathrm{~V}$ & $\begin{array}{l}\text { Calculated maximum value of the noise } \\
\text { voltage at the LNLFA input resulting from the } \\
\text { local oscillator amplitude noise }\end{array}$ \\
\hline$V_{\mathrm{nAO}}$ & $\mathrm{V}$ & $\begin{array}{l}\text { Calculated maximum value of the noise } \\
\text { voltage at the ADC input resulting from the } \\
\text { local oscillator amplitude noise }\end{array}$ \\
\hline$P_{\mathrm{nLF}}$ & $\mathrm{W}$ & $\begin{array}{l}\text { Calculated noise power related to the LNLFA } \\
\text { input resulting from the noise of LNLFA }\end{array}$ \\
\hline$V_{\mathrm{nLF}}$ & $\mathrm{V}$ & $\begin{array}{l}\text { Calculated noise voltage related to the LNLFA } \\
\text { input resulting from the noise of LNLFA }\end{array}$ \\
\hline$V_{\mathrm{nLFO}}$ & $\mathrm{V}$ & $\begin{array}{l}\text { Calculated noise voltage related to the ADC } \\
\text { input resulting from the noise of LNLFA }\end{array}$ \\
\hline$P_{\mathrm{nT}}$ & $\mathrm{W}$ & $\begin{array}{l}\text { Calculated maximum total noise power at the } \\
\text { LNLFA input }\end{array}$ \\
\hline$V_{\mathrm{nT}}$ & $\mathrm{V}$ & $\begin{array}{l}\text { Calculated maximum total noise voltage at the } \\
\text { LNLFA input }\end{array}$ \\
\hline$V_{\mathrm{nTO}}$ & $\mathrm{V}$ & $\begin{array}{l}\text { Calculated maximum total noise voltage at the } \\
\text { ADC input }\end{array}$ \\
\hline$V_{\mathrm{nMO} \_\mathrm{I}}$ & $\mathrm{V}$ & $\begin{array}{l}\text { Measured maximum total noise voltage at the } \\
\text { ADC input - I channel }\end{array}$ \\
\hline$V_{\mathrm{nMO} \_\mathrm{Q}}$ & $\mathrm{V}$ & $\begin{array}{l}\text { Measured maximum total noise voltage at the } \\
\text { ADC input }- \text { Q channel }\end{array}$ \\
\hline
\end{tabular}

\section{References}

[1] DICKMANN, J., KLAPPSTEIN, J., HAHN, M., et al. Automotive radar the key technology for autonomous driving: From detection and ranging to environmental understanding. In 2016 IEEE Radar Conference. Philadelphia (PA, USA), 2016, p. 1-6. DOI: 10.1109/RADAR.2016.7485214

[2] MEINEL, H., DICKMANN, J. Automotive radar: From its origins to future directions. Microwave Journal, 2013, vol. 56, no. 9, p. 24-40.

[3] FEGER, R., HADERER, A., STELZER, A. Experimental verification of a 77-GHz synthetic aperture radar system for automotive applications. In 2017 IEEE MTT-S International Conference on Microwaves for Intelligent Mobility (ICMIM). Nagoya (Japan), 2017, p. 111-114. DOI: 10.1109/ICMIM.2017.7918869

[4] SWAMI, P., JAIN, A., GOSWAMI, P., et al. High performance automotive radar signal processing on TI's TDA3X platform. In 2017 IEEE Radar Conference (RadarConf). Seattle (WA, USA), 2017, p. 1317-1320. DOI: 10.1109/RADAR.2017.7944409

[5] NGUYEN, D., YAMADA, S., PARK, B. K., et al. Noise considerations for remote detection of life signs with microwave Doppler radar. In 2007 29th Annual International Conference of the IEEE Engineering in Medicine and Biology Society. Lyon (France), 2007, p. 1667-1670. DOI: 10.1109/IEMBS.2007.4352628

[6] CHIOUKH, L., BOUTAYEB, H., DESLANDES, D., et al. Noise and sensitivity of harmonic radar architecture for remote sensing and detection of vital signs. IEEE Transactions on Microwave 
Theory and Techniques, vol. 62, no. 9, p. 1847-1855, 2014. DOI: 10.1109/TMTT.2014.2343934

[7] NANZER, J. A. A review of microwave wireless techniques for human presence detection and classification. IEEE Transactions on Microwave Theory and Techniques, 2017, vol. 65, no. 5, p. 1780-1794. DOI: 10.1109/TMTT.2017.2650909

[8] WANG, G., GU, C., INOUE, T., et al. A hybrid FMCWinterferometry radar for indoor precise positioning and versatile life activity monitoring. IEEE Transactions on Microwave Theory and Techniques, 2014, vol. 62, no. 11, p. 2812-2822. DOI: 10.1109/TMTT.2014.2358572

[9] HSU, M. L., LIU, T. H., YANG, T. C., et al. Bee searching radar with high transmit-receive isolation using pulse pseudorandom code. IEEE Transactions on Microwave Theory and Techniques, 2016, vol. 64, no. 12, p. 4324-4335. DOI: 10.1109/TMTT.2016.2613531

[10] SIDDIQ, K., WATSON, R. J., PENNOCK, S. R., et al. Phase noise analysis in FMCW radar systems. In 2015 European Radar Conference (EuRAD). Paris (France), 2015, p. 501-504. DOI: 10.1109/EuRAD.2015.7346347

[11] SIDDIQ, K., WATSON, R. J., PENNOCK, S. R., et al. Analysis of sampling clock phase noise in homodyne FMCW radar systems. In 2016 IEEE Radar Conference (RadarConf). Philadelphia (PA, USA), 2016, p. 1-4. DOI: 10.1109/RADAR.2016.7485302

[12] DUDEK, M., NASR, I., KISSINGER, D., et al. The impact of phase noise parameters on target signal detection in FMCW-radar system simulations for automotive applications. In Proceedings of 2011 IEEE CIE International Conference on Radar. Chengdu (China), 2011, p. 494-497. DOI: 10.1109/CIE-Radar.2011.6159587

[13] BUDGE Jr., M. C., BURT, M. P. Range correlation effects in radars. In Record of the 1993 IEEE National Radar Conference. Lynnfield (MA, USA), 1993, p. 212-216. DOI: 10.1109/NRC.1993.270463

[14] BUDGE, M. C., BURT, M. P. Range correlation effects on phase and amplitude noise. In Proceedings of IEEE Southeastcon '93, Charlotte (NC, USA), 1993, p. 1-5. DOI: 10.1109/SECON.1993.465731

[15] JENIK, V., HUDEC, P., PANEK, P. Noise parameters of CW radar sensors used in active defence systems, Radioengineering, 2011, vol. 21, no. 2, p. 632-639. ISSN: 1210-2512

[16] GHEEN, K., AGILENT TECHNOLOGIES, Phase Noise Measurement Methods and Techniques. 48 pages. [Online] Cited 201603-01. Available at: http://www.keysight.com/upload/cmc_upload /All/PhaseNoise_webcast_19Jul12.pdf?\&cc $=\mathrm{CZ \& lc=eng}$

[17] VISCOR, I., HALAMEK, J. Phase noise measurement. In Proceedings of the Radioelektronika 2001. Brno (Czech Republic), 2001, p. 131-134.

[18] WAN, K. W., AUSTIN, J., VILAR, E. A novel approach to the simultaneous measurement of phase and amplitude noise of oscillators. In Proceedings of the 44th Annual Symposium on Frequency Control. Baltimore (MD, USA), 1990, p. 140-144. DOI: 10.1109/FREQ.1990.177491

[19] RUPPALT, L. B., McKINSTRY, D. R., LAURITZEN, K. C., et al. Simultaneous digital measurement of phase and amplitude noise. In IEEE International Frequency Control Symposium (FCS).
Newport Beach (CA, USA), 2010, p. 97-102. DOI: 10.1109/FREQ.2010.5556365

[20] ÁVILA-RUIZ, J. M., MOSCOSO-MÁRTIR, A., DURÁNVALDEIGLESIAS, E., et al. Six-port-based architecture for phase noise measurement in the UWB band. Journal of Electrical and Computer Engineering, 2014, Article ID 646738, p. 1-10. DOI: $10.1155 / 2014 / 646738$

[21] GHEIDI, H., BANAI, A. Phase-noise measurement of microwave oscillators using phase-shifterless delay-line discriminator. IEEE Transactions on Microwave Theory and Techniques, 2010, vol. 58, no. 2, p. 468-477. DOI: 10.1109/TMTT.2009.2038452

[22] GHEIDI, H., BANAI, A. Phase-shifterless phase-noise measurement of microwave oscillators using high-Q cavity frequency discriminator. In The 81st Microwave Measurement Conference (ARFTG). Seattle (WA, USA), 2013, p. 1-3. DOI: 10.1109/ARFTG.2013.6579027

[23] RUBIOLA, E. The measurement of AM noise of oscillators. In International Frequency Control Symposium and Exposition 2006. Miami (FL, USA), 2006, p. 750-758. DOI: 10.1109/FREQ.2006.275483

\section{About the Authors ...}

Vojtech JENIK received his M.Sc. in Telecommunications and Radio Engineering from the Czech Technical University in Prague in 2010. He is a PhD. student at the Dept. of Electromagnetic Field. His research interests include microwave sensors for the detection of high-speed moving objects and other microwave measurement methods.

Premysl HUDEC received his M.S. degree in Electrical Engineering from the Czech Technical University in Prague in 1982, and his Ph.D. Degree in 1995. Since 2010, he has been working as an Associated Professor at its Dept. of Electromagnetic Field. His teaching duties include CAD of microwave circuits and microwave measurements. In recent years, his research activities have been focused on the employment of microwave technology in security applications. Primarily, these works include the design of different types of microwave radar sensors for active defense systems and the development of RFID systems for long-range identification.

Petr PANEK received his M.Sc. and Ph.D. degrees in Electrical Engineering from the Czech Technical University in Prague, Czech Republic in 1982 and 1988, respectively. He is with the Institute of Photonics and Electronics, Academy of Sciences of the Czech Republic, Prague. He has vast experience in industrial R\&D. His main research interests are time measurement and radio positioning. 